\title{
SIR-OPTIMIZED DFT-BANK TRANSCEIVERS FOR MULTIPATH FADING CHANNELS
}

\author{
See-May Phoong, Yubing Chang, Chun-Yang Chen \\ Dept. of EE \& Grad. Inst. of Comm. Engr. \\ National Taiwan Univ. \\ Taipei, Taiwan, ROC
}

\begin{abstract}
In this paper, we study DFT-bank transceivers with filter length longer than the block size. We show how to optimize the DFT-bank transceiver so that both the intra-band and cross-band interferences are minimized for unknown multipath channels. No costly post processing technique is needed and the only channel dependent part is a set of one-tap equalizers at the receiver. The optimization problem is formulated as a Rayleigh-Ritz ratio whose solution is well-known. Experiments are carried out for transmission over random multipath channels and the results show that satisfactory SIR performance can be obtained.
\end{abstract}

\section{INTRODUCTION}

The OFDM (orthogonal frequency division multiplexing) and DMT (discrete multitone) techniques have found many applications in both wireless and wired transmissions [1]. These systems have low complexity and can effectively combat intersymbol interference (ISI). However the DFT filters suffer from very poor frequency responses. The stopband attenuation is only $13 \mathrm{~dB}$ and it decays at a rate of $1 / f$ only. In many applications, it is often desirable to have filters with better frequency responses.

Many solutions have been proposed for the design of transceivers with better transmitting and receiving filters. In particular, the filter bank approach [2] [3] [4] has drawn many attentions recently. In [2], the authors propose the so-called discrete wavelet multitone (DWMT) system, in which perfect reconstruction (PR) filter bank is used as the transceiver. For multipath channels, there is intraband as well as cross-band interference in these transceivers. Unlike the OFDM system, there is no simple equalization technique for DWMT systems. Comparisons and performance evaluations of these filter bank transceivers have been conducted in [3]. The results show that though the filter bank has near PR property, the ISI introduced by the channel can seriously degrade the system performance. To reduce the amount of ISI, intra- and cross-band equalization are performed on the receiver outputs in [2]. Filter bank transceivers that enjoy the ISI-free property for unknown multipath channels have been studied in [4]. By judiciously placing the zeros of the transmitting filters, the authors in [4] showed that the proposed AMOUR transceiver can obtain ISI-free transmission by using a Vandermonde matrix at the receiver. However, the AMOUR transceiver belongs to the class of block transmission schemes, i.e. transmitting filter length restricted to the block size. Moreover

This work was supported in parts by National Science Council, Taiwan, ROC, under NSC 92-2219-E-002-015 and 92-2213-E-009-022, Ministry of Education, Taiwan, ROC, under Grant \# 89E-FA06-2-4, and the Lee and MTI Center for Networking Research.

\author{
Yuan-Pei Lin \\ Dept. Electrical and Control Engr. \\ National Chiao Tung Univ. \\ Hsinchu, Taiwan, ROC
}

there is no simple method to design AMOUR transceivers with good frequency responses.

In this paper, we design DFT-bank transceivers for multipath fading channels. Although the proposed transceiver belongs to the class of overlapping-block transmission, the only channel dependent part is a set of one-tap equalizers at the receiver, like the OFDM system. For a set of good receiving filters, the transmitting prototype filter can be optimized so that SIR (signal-tointerference ratio) is maximized. Conversely, we can also design the receiving prototype filter to maximize SIR given transmitting filters. We show that such an optimization problem can be formulated as a Rayleigh-Ritz ratio, whose solution is well known [5]. Simulation results show that DFT-bank transceivers with satisfactory SIR value can be obtained.

\section{ISI-FREE DFT-BANK TRANSCEIVERS}

Fig. 1 shows a filter bank transceiver. The number of bands is $M$ whereas the up/downsampling factor is $N$. We assume that $N \geq M$. The number of redundancy samples is $N-M$. We consider only DFT-bank transceiver where the transmitting filters $F_{k}(z)$ and the receiving filters $H_{k}(z)$ are DFT modulated versions of the prototypes $F_{0}(z)$ and $H_{0}(z)$ respectively. Their relation is given by

$F_{k}(z)=F_{0}\left(z W^{k}\right), H_{k}(z)=H_{0}\left(z W^{k}\right)$, where $W=e^{-j \frac{2 \pi}{M}}$

for $1 \leq k \leq M-1$. Let $n_{f}$ and $n_{h}$ be respectively the orders of $F_{0}(z)$ and $H_{0}(z)$. In this paper, both $n_{f}$ and $n_{f}$ can be larger than $N$. The advance element $z^{l_{0}}$, where $l_{0}$ is an arbitrary integer, is added at the receiver for the convenience of discussion. We assume that the transmission channel does not vary rapidly so that it can be modeled as an LTI channel with transfer function $C(z)$, as shown in Fig. 1. Let $L$ be the channel order so that $C(z)=$ $\sum_{n=0}^{L} c(n) z^{-n}$.

Using multirate identities, one can verify from Fig. 1 that the transfer function from $x_{i}(n)$ to $\hat{x}_{j}(n)$ is an LTI system:

$$
\begin{aligned}
T_{j i}(z) & =\left[F_{i}(z) C(z) z^{l_{0}} H_{j}(z)\right]_{\downarrow N} \\
& =\sum_{l=0}^{L} c(l)\left[F_{i}(z) H_{j}(z) z^{l_{0}-l}\right]_{\downarrow N},
\end{aligned}
$$

where $[\bullet]_{\downarrow N}$ denotes the $N$-fold downsampling operation. From the above equation, it is clear that the DFT-bank transceiver is ISIfree for unknown multipath channels if

$$
T_{j i}(z)=G_{i} z^{-n_{0}} \delta(j-i) .
$$




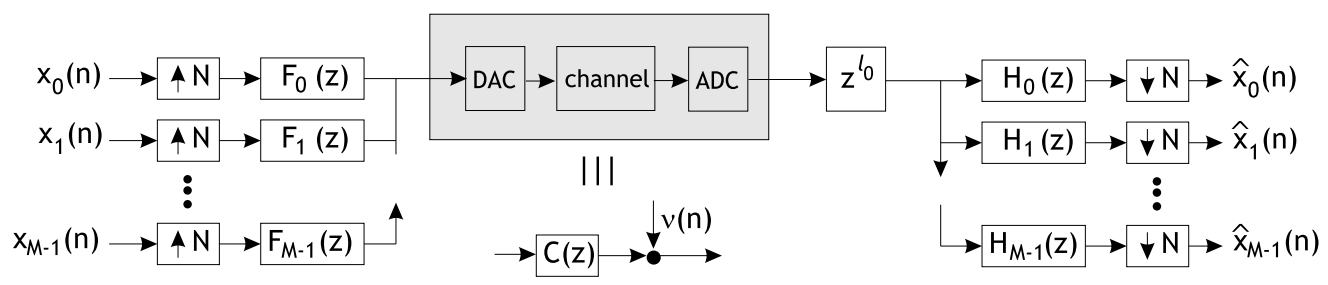

Figure 1: A filter bank transceiver.

Because $l_{0}$ is an arbitrary integer, we can assume $n_{0}=0$ without any loss of generality. In this paper, we set $n_{0}=0$. From (2) and (3), one can immediately conclude that interchanging the roles of transmitting and receiving filters does not change the ISI-free property. Define the sequences $\alpha_{i, l}(n)$ and $\beta_{i, j, l}(n)$ as:

$$
\left[F_{i}(z) H_{j}(z) z^{l_{0}-l}\right]_{\downarrow N}= \begin{cases}\alpha_{i, l}(0)+\sum_{n, n \neq 0} \alpha_{i, l}(n) z^{-n} & i=j, \\ \sum_{n} \beta_{i, j, l}(n) z^{-n} & i \neq j,\end{cases}
$$

for $0 \leq i, j \leq M-1$ and $0 \leq l \leq L$. Note that $\beta_{i, j, l}(n)$ is not defined for $i \neq j$. For convenience, we define

$$
\beta_{i, i, l}(n)=0, \text { for all } i, l, n .
$$

Using these sequences, the ISI-free condition can be rewritten as

$$
\alpha_{i, l}(n)=\alpha_{i, l}(0) \delta(n), \beta_{i, j, l}(n)=0 \text { for all } i, j, l, n .
$$

Whenever we have $\alpha_{i, l}(n) \neq 0$ for $n \neq 0$ or $\beta_{i, j, l}(n) \neq 0$, then $\alpha_{i, l}(n)$ and $\beta_{i, j, l}(n)$ contribute respectively to the intra-band and the cross-band intersymbol interference.

Recall that the transmitting and receiving filters are DFT modulated versions of prototype filters. Using this fact, one can show the following lemma (see [6] for a proof).

Lemma 1 For DFT modulated filter bank transceivers with filters defined in (1), the sequences $\alpha_{i, l}(n)$ and $\beta_{i, j, l}(n)$ defined in (4) satisfy

$$
\begin{aligned}
\alpha_{i, l}(n) & =W^{-i\left(l_{0}-l+n\right)} \alpha_{0, l}(n) \\
\beta_{i, j, l}(n) & =W^{-i\left(l_{0}-l+n\right)} \beta_{0,((j-i))_{M}, l}(n),
\end{aligned}
$$

where $((j-i))_{M}$ represents $(j-i)$ modulo $M$.

Using Lemma 1, the ISI-free condition can be further simplified as:

$$
\alpha_{0, l}(n)=\alpha_{0, l}(0) \delta(n), \beta_{0, j, l}(n)=0, \text { for all } j, l, n .
$$

When the transceiver achieves the ISI-free condition, any frequency selective channel $c(n)$ with order $\leq L$ is converted into a set of $M$ parallel frequency nonselective subchannels. The gain of the $i$ th subchannel is given by

$$
G_{i}=\sum_{l=0}^{L} c(l) \alpha_{i, l}(0)=W^{-i l_{0}} \sum_{l=0}^{L} \alpha_{0, l}(0) W^{i l} c(l) .
$$

Note that $\alpha_{0, l}(n)$ and $\beta_{0, j, l}(n)$ are functions of the prototype filters $h_{0}(n)$ and $f_{0}(n)$. Using (4), one can write

$$
\left[\begin{array}{c}
\alpha_{0,0}(n) \\
\alpha_{0,1}(n) \\
\vdots \\
\alpha_{0, L}(n)
\end{array}\right]=\mathbf{A}(n)\left[\begin{array}{c}
f_{0}(0) \\
f_{0}(1) \\
\vdots \\
f_{0}\left(n_{f}\right)
\end{array}\right]
$$

$$
\left[\begin{array}{c}
\beta_{0, j, 0}(n) \\
\beta_{0, j, 1}(n) \\
\vdots \\
\beta_{0, j, L}(n)
\end{array}\right]=\mathbf{B}_{j}(n)\left[\begin{array}{c}
f_{0}(0) \\
f_{0}(1) \\
\vdots \\
f_{0}\left(n_{f}\right)
\end{array}\right],
$$

where $\mathbf{A}(n)$ and $\mathbf{B}_{j}(n)$ are $(L+1)$ by $\left(n_{f}+1\right)$ matrices and their entries consist of the impulse response $h_{0}(n)$. The exact forms of these matrices are given in the Appendix B of [6]. Define the vectors

$$
\mathbf{f}_{0} \triangleq\left[\begin{array}{c}
f_{0}(0) \\
f_{0}(1) \\
\vdots \\
f_{0}\left(n_{f}\right)
\end{array}\right], \quad \boldsymbol{\alpha} \triangleq\left[\begin{array}{c}
\alpha_{0,0}(0) \\
\alpha_{0,1}(0) \\
\vdots \\
\alpha_{0, L}(0)
\end{array}\right]
$$

Then the ISI-free conditions in (5) can be written as

$$
\mathbf{A}(n) \mathbf{f}_{0}=\boldsymbol{\alpha} \delta(n), \mathbf{B}_{j}(n) \mathbf{f}_{0}=\mathbf{0} .
$$

\section{DESIGN OF DFT-BANK TRANSCEIVERS}

In many applications, it is often desired to have transmitting filters or receiving filters with good frequency responses. Depending on applications, our design problem is either (1) given a good lowpass transmitting prototype filter $F_{0}(z)$, design the receiving prototype filter $H_{0}(z)$ to achieve the ISI-free property or SIR maximization, or (2) given a good lowpass receiving prototype filter $H_{0}(z)$, design the transmitting prototype filter $F_{0}(z)$ to achieve the ISI-free property or SIR maximization. As interchanging the roles of the transmitting and receiving filters does not affect the ISI-free property, Problem 1 can be easily formulated into Problem 2, and vice verse. In this paper, we will study Problem 2 only. Hence in the rest of the paper, $H_{0}(z)$ is a predetermined good lowpass filter.

\subsection{Least-Squares Solution}

Recall the ISI-free condition given in (6). For a fixed $H_{0}(z)$, our goal is to design $F_{0}(z)$ so that (6) is satisfied. One way to solve this problem is to use the least-squares method. One can write the conditions in (6) as a single matrix equation:

$$
\boldsymbol{\Psi} \mathbf{f}_{0}=\left[\begin{array}{c}
\boldsymbol{\alpha} \\
\mathbf{0}
\end{array}\right]
$$

When the desired parameters $\boldsymbol{\alpha}$ are known, one can use the leastsquares method to solve the above linear equations and obtain

$$
\mathbf{f}_{0, L S}=\left(\boldsymbol{\Psi}^{\dagger} \boldsymbol{\Psi}\right)^{-1} \boldsymbol{\Psi}^{\dagger}\left[\begin{array}{c}
\boldsymbol{\alpha} \\
\mathbf{0}
\end{array}\right]
$$


In many applications, it is desired to have transceivers that maximize SIR. In this least-squares approach, the optimal $\alpha_{0, l}(0)$ that maximizes SIR is not known.

\subsection{SIR-Optimized Solution}

In the following derivation, we will assume that the input signals $x_{i}(n)$ satisfy

$$
E\left\{x_{i}(n) x_{j}^{*}(m)\right\}=\mathcal{E}_{x} \delta(i-j) \delta(n-m),
$$

where $\mathcal{E}_{x}$ is the symbol energy. This mild assumption can be satisfied by properly interleaving the input data. We will consider two cases: (i) known channel and (ii) multipath fading channel.

Known Channels: In this case, the impulse response $c(n)$ is known. One can write the output of the $j$ th subchannel as

$$
\begin{aligned}
\hat{x}_{j}(n)= & {\left[\sum_{l=0}^{L} \alpha_{j, l}(0) c(l)\right] x_{j}(n)+\sum_{l=0}^{L} c(l)\left[\alpha_{j, l}(n)-\right.} \\
& \left.\alpha_{j, l}(0) \delta(n)\right] * x_{j}(n)+\sum_{i=0}^{M-1} \sum_{l=0}^{L} c(l) \beta_{i, j, l}(n) * x_{i}(n),
\end{aligned}
$$

where $*$ denotes convolution. The three summation terms on the right hand side are respectively the desired signal, the intra-band and cross-band intersymbol interferences. Using (8), one can express the signal and interference powers at the $j$ th subchannel as

$$
\begin{gathered}
P_{s i g}(j)=\mathcal{E}_{x} \underbrace{\left|\sum_{l=0}^{L} \alpha_{j, l}(0) c(l)\right|^{2}}_{\phi_{0}(j)} \\
P_{i s i}(j)=\mathcal{E}_{x}(\underbrace{\sum_{n, n \neq 0}\left|\sum_{l=0}^{L} \alpha_{j, l}(n) c(l)\right|^{2}}_{\phi_{1}(j)}+\underbrace{\sum_{i, n}\left|\sum_{l=0}^{L} \beta_{i, j, l}(n) c(l)\right|^{2}}_{\phi_{2}(j)}) .
\end{gathered}
$$

The SIR expression is given by

$$
S I R_{c}=\frac{\sum_{j=0}^{M-1} \phi_{0}(j)}{\sum_{j=0}^{M-1} \phi_{1}(j)+\sum_{j=0}^{M-1} \phi_{2}(j)},
$$

Using the results in Lemma 1, we can write

$$
\sum_{j=0}^{M-1} \phi_{0}(j)=\sum_{j=0}^{M-1}\left|\sum_{l=0}^{L} W^{j l} \alpha_{0, l}(0) c(l)\right|^{2}=\left\|\mathbf{W}_{L+1} \mathbf{D}_{c} \boldsymbol{\alpha}\right\|^{2}
$$

where $\|\mathbf{v}\|$ denotes the 2-norm of $\mathbf{v}$. The diagonal matrix $\mathbf{D}_{c}=$ $\operatorname{diag}[c(0) c(1) \ldots c(L)]$ and $\mathbf{W}_{L+1}$ is the $M$ by $(L+1)$ matrix formed by the first $(L+1)$ columns of the DFT matrix $\mathbf{W}$. Substituting $\boldsymbol{\alpha}=\mathbf{A}(0) \mathbf{f}_{0}$ into the above equation, we get

$$
\sum_{j=0}^{M-1} \phi_{0}(j)=\mathbf{f}_{0}^{\dagger}(\underbrace{\mathbf{A}^{\dagger}(0) \mathbf{D}_{c}^{\dagger} \mathbf{W}_{L+1}^{\dagger} \mathbf{W}_{L+1} \mathbf{D}_{c} \mathbf{A}(0)}_{\mathbf{Q}_{0}}) \mathbf{f}_{0} .
$$

Similarly it can be verified that we can write

$$
\sum_{j=0}^{M-1} \phi_{1}(j)=\mathbf{f}_{0}^{\dagger} \mathbf{Q}_{1} \mathbf{f}_{0} \quad \sum_{j=0}^{M-1} \phi_{2}(j)=\mathbf{f}_{0}^{\dagger} \mathbf{Q}_{2} \mathbf{f}_{0},
$$

where

$$
\begin{aligned}
& \mathbf{Q}_{1}=\sum_{n, n \neq 0} \mathbf{A}^{\dagger}(n) \mathbf{D}_{c}^{\dagger} \mathbf{W}_{L+1}^{\dagger} \mathbf{W}_{L+1} \mathbf{D}_{c} \mathbf{A}(n) \\
& \mathbf{Q}_{2}=\sum_{n} \sum_{j=1}^{M-1} \mathbf{B}_{j}^{\dagger}(n) \mathbf{D}_{c}^{\dagger} \mathbf{W}_{L+1}^{\dagger} \mathbf{W}_{L+1} \mathbf{D}_{c} \mathbf{B}_{j}(n) .
\end{aligned}
$$

Using these results, we can write SIR as

$$
S I R_{c}=\frac{\mathbf{f}_{0}^{\dagger} \mathbf{Q}_{0} \mathbf{f}_{0}}{\mathbf{f}_{0}^{\dagger}\left(\mathbf{Q}_{1}+\mathbf{Q}_{2}\right) \mathbf{f}_{0}} .
$$

Since $\mathbf{Q}_{i}$ is positive definite (except for some rare cases), the above SIR expression is a Rayleigh-Ritz ratio [5]. The optimal $\mathbf{f}_{0}$ can be found by solving an eigen problem.

Multipath Fading Channels: In many applications, $c(n)$ might not be available and we may have only the statistics of the transmission channels. Consider multipath fading channels with $(L+1)$ taps $c(l)$ for $0 \leq l \leq L$. Assume that $c(l)$ are complex random variables that satisfy

$$
E\{c(l)\}=0, \quad E\left\{c(l) c^{*}(l-k)\right\}=\sigma_{l}^{2} \delta(k),
$$

for $0 \leq l \leq L$. In this case, we consider average powers. Taking the expectation of (9) and (10) with respect to $c(l)$ and using Lemma 1 to simplify the results, we get

$$
\bar{P}_{s i g}(j)=\mathcal{E}_{x} \underbrace{\sum_{l=0}^{L}\left|\alpha_{0, l}(0)\right|^{2} \sigma_{l}^{2}}_{\zeta_{0}(j)}
$$

$$
\bar{P}_{i s i}(j)=\mathcal{E}_{x}(\underbrace{\sum_{n, n \neq 0} \sum_{l=0}^{L}\left|\alpha_{0, l}(n)\right|^{2} \sigma_{l}^{2}}_{\zeta_{1}(j)}+\underbrace{\sum_{i, n} \sum_{l=0}^{L}\left|\beta_{0, i, l}(n)\right|^{2} \sigma_{l}^{2}}_{\zeta_{2}(j)}) .
$$

The average SIR is therefore given by

$$
\overline{S I R}=\frac{\sum_{j=0}^{M-1} \zeta_{0}(j)}{\sum_{j=0}^{M-1} \zeta_{1}(j)+\sum_{j=0}^{M-1} \zeta_{2}(j)} .
$$

Similar to the previous case, one can verify that

$$
\begin{gathered}
\sum_{j=0}^{M-1} \zeta_{0}(j)=\mathbf{f}_{0}^{\dagger} \mathbf{A}^{\dagger}(0) \mathbf{D}_{\sigma} \mathbf{A}(0) \mathbf{f}_{0} \\
\sum_{j=0}^{M-1} \zeta_{1}(j)=\mathbf{f}_{0}^{\dagger}\left(\sum_{n, n \neq 0} \mathbf{A}^{\dagger}(n) \mathbf{D}_{\sigma} \mathbf{A}(n)\right) \mathbf{f}_{0} \\
\sum_{j=0}^{M-1} \zeta_{2}(j)=\mathbf{f}_{0}^{\dagger}\left(\sum_{n} \sum_{i=1}^{M-1} \mathbf{B}_{i}^{\dagger}(n) \mathbf{D}_{\sigma} \mathbf{B}_{i}(n)\right) \mathbf{f}_{0},
\end{gathered}
$$

where $\mathbf{D}_{\sigma}$ is the diagonal matrix $\operatorname{diag}\left[\begin{array}{llll}\sigma_{0}^{2} & \sigma_{1}^{2} & \ldots & \sigma_{L}^{2}\end{array}\right]$. From the above expressions, one can clearly see that the average SIR can also be formulated into a Rayleigh-Ritz ratio. The optimal $\mathbf{f}_{0}$ can be obtained by solving a corresponding eigen problem. When no channel information is available, the channel is often modeled as iid channels. In this case, the results can be obtained by setting $\sigma_{i}=1$ and the transceiver becomes channel independent. 
The Choice of $z^{l_{0}}$ : Note that $\mathbf{A}(n)$ and $\mathbf{B}_{i}(n)$ depend on the choice of the integer $l_{0}$. One has to search for the best $l_{0}$ to maximize either $S I R_{c}$ or $\overline{S I R}$. In our simulations, we find that the best $l_{0}$ always falls within the range of $-N / 2 \leq l_{0}-\left(n_{f}-n_{h}\right) / 2<$ $N / 2$.

\section{SIMULATIONS}

In the examples, the receiving prototype filter $h_{0}(n)$ is a unit norm lowpass filter designed using the eigenfilter method. The coefficients $h_{0}(n)$ are designed to minimize $\int_{\omega_{s}}^{\pi}\left|H_{0}\left(e^{j \omega}\right)\right|^{2} d \omega$. The channels $c(n)$ are multipath fading channels. The coefficients $c(n)$ are independent circular complex Gaussian random variables with variances $\sigma_{n}^{2}$. We have used 10,000 random channels in the experiments. All the SIR values shown in the plots are computed by taking the average value over 10,000 random channels.

Example 1. In this example, we design transceiver with $M=64$ and $N=80$. The receiving prototype filter $H_{0}(z)$ has $n_{h}=160$, $\omega_{s}=0.033 \pi$ and a stopband attenuation of more than $61 \mathrm{~dB}$. The random channels are iid channels with $(L+1)$ taps. The SIR curves for $L=4,8,16$ are shown in Fig. 2. We can obtain a moderate SIR value even when the receiving filter has a $61 \mathrm{~dB}$ stopband attenuation.

Example 2. We take $M=16, N=20$ and $L=4$. The receiving filter prototype $H_{0}(z)$ has $n_{h}=40, \omega_{s}=0.13 \pi$ and a stopband attenuation of $61 \mathrm{~dB}$. The random channels have an exponentially decay delay profile and $\sigma_{n}^{2}=\frac{32}{31} 2^{-n}$. We consider 3 scenarios: (i) the channel impulse response $c(n)$ is known; (ii) only $\sigma_{n}^{2}$ is known; and (iii) no channel information is available and we assume iid channels. In Case (i), we design an optimal transceiver for each of the 10,000 random channels, whereas in Cases (ii) and (iii), we design only one optimal transceiver. The cost of designing optimal transceivers for known channels is significantly higher. The results are shown in Fig. 3. As we might expect, if $c(n)$ is known, the transceiver will have the best SIR performance. Comparing Case (i) and Case (ii), the improvement is not significant. If we compare Case (ii) and (iii), we can obtain a moderate gain. Hence incorporating the channel profile in the design can significantly increase the SIR performance.

\section{CONCLUSIONS}

In this paper, we study DFT-bank transceivers with filter length longer than the block size. Given a fixed receiving (or transmitting) prototype filter, we have shown that the problem of finding the best transmitting (or correspondingly receiving) prototype filter that maximizes the SIR can be formulated as a Rayleigh-Ritz ratio. Though our discussion is restricted to the case of DFT modulated filters, the results can be generalized to the case of non modulated filters. Moreover, in some cases there is no loss of generality in assuming that the optimal transmitting filters are DFT modulated version of a prototype filter [6].

\section{REFERENCES}

[1] R. van Nee and R. Prasad, OFDM for wireless multimedia communications, Artech House, Boston, 2000.

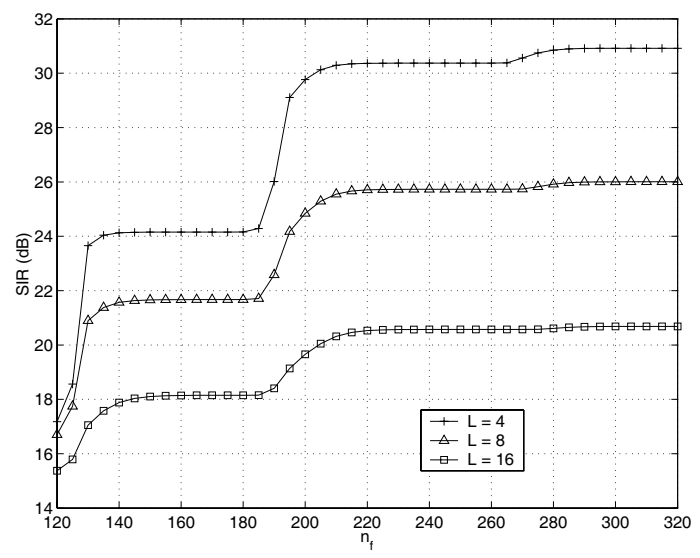

Figure 2: SIR optimized 64-band transceivers.

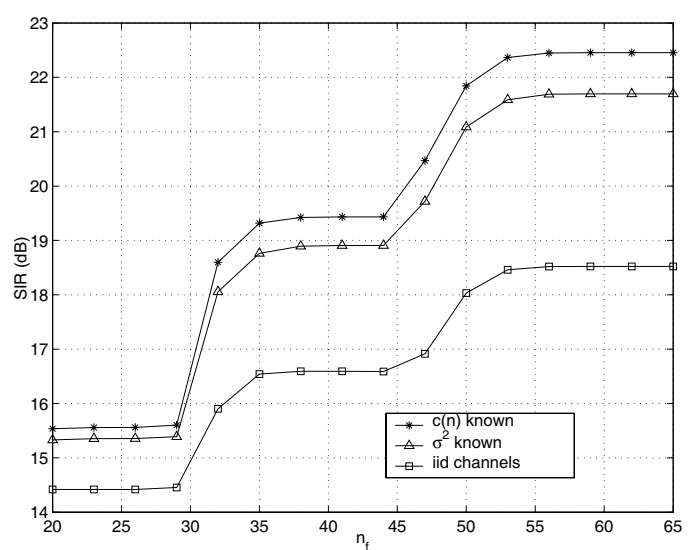

Figure 3: Performance of transceivers designed using different degrees of channel profile.

[2] S. D. Sandberg and M. A. Tzannes, "Overlapped Discrete Multitone Modulation for High Speed Copper Wire Communications," IEEE J. SAC, Dec. 95.

[3] A. D. Rizos, J. G. Proakis, and T. Q. Nguyen, "Comparison of DFT and Cosine Modulated Filter Banks in Multicarrier Modulation," IEEE Global Telecommunications Conference, pp. 687-691, 1994.

[4] G. B. Giannakis, Z. Wang, A. Scaglione, and S. Barbarossa, "AMOUR - Generalized Multicarrier Transceivers for Blind CDMA Regardless of Multipath," IEEE Trans. Comm., vol. 48, no. 12, Dec. 2000.

[5] R. A. Horn and C. R. Johnson, Matrix Analysis, Cambridge University Press, 1985.

[6] S. Phoong, Y. Chang, C. Chen and Y. Lin, "DFT modulated filter bank transceivers for multipath fading channels," submitted to IEEE Trans. SP, Aug. 2003. 